\title{
Biomarcadores del estrés oxidativo y su asociación con la severidad clínica de pacientes venezolanos con síndrome drepanocítico.
}

\author{
Diego Higuera ${ }^{1,2}$, Edwina Hergueta ${ }^{3}$, Narley Estepa ${ }^{3}$, Emelyn Salazar ${ }^{1,4}$, Carlos Ibarra ${ }^{1}$, \\ Karin Péres ${ }^{1}$, Belsy Guerrero ${ }^{1}$, Martha Bravo ${ }^{5}$ y Ana María Salazar ${ }^{1}$ \\ ${ }^{1}$ Laboratorio de Fisiopatología, Sección Coagulación, Centro de Medicina Experimental, \\ Instituto Venezolano de Investigaciones Científicas. Caracas, Venezuela. \\ ${ }^{2}$ Laboratorio de Hemoglobinopatías y Talasemias, Banco Municipal de Sangre del \\ Distrito Capital, Venezuela.Caracas, Venezuela. \\ ${ }^{3}$ Escuela de Bioanálisis, Facultad de Medicina, Universidad Central de Venezuela. \\ Caracas, Venezuela. \\ ${ }^{4}$ National Natural Toxins Research Center (NNTRC), Texas A \& M University, Kingssille, USA. \\ ${ }^{5}$ Laboratorio de Investigación de Hemoglobinas Anormales, Instituto Anatómico "José \\ Izquierdo", Universidad Central de Venezuela. Caracas, Venezuela.
}

Palabras clave: Hemoglobina S; síndrome drepanocítico; estrés oxidativo; severidad clínica.

Resumen. El síndrome drepanocítico (SD) comprende un grupo de anemias hemolíticas caracterizadas por episodios de dolor agudo y daño progresivo de diferentes órganos. Es una de las alteraciones hereditarias más comunes en el mundo y, en Venezuela, se ha descrito una frecuencia entre 0 y $7 \%$. La fisiopatología del SD se basa en la presencia de moléculas de hemoǵlobina (Hb) estructuralmente anormales ( $\mathrm{HbS}$ ), que polimerizan en estado desoxigenado e inducen la aparición de crisis vaso-oclusivas y hemolíticas. La hiperproducción de especies reactivas de oxígeno y de nitrógeno, forman parte de la fisiopatología, e inducen un estado de estrés oxidativo que se asocia con disfunción endotelial y daño tisular. En 23 personas aparentemente sanas y 22 pacientes con SD clasificados según genotipo (HbSS, HbSG y HbSßtal) y fenotipo (leve, moderado y severo), se evaluaron biomarcadores de estrés oxidativo (nitritos séricos, dienos conjugados, sustancias reactivas al ácido tiobarbitúrico (TBARS) y homocisteína (Hey) y su asociación con la severidad clínica. Los resultados mostraron un aumento significativo de TBARS en los pacientes HbSS con fenotipo severo,

Autor de Correspondencia: Ana María Salazar. Laboratorio de Fisiopatología-Sección Coagulación, Centro de Medicina Experimental, Instituto Venezolano de Investigaciones Científicas. Caracas, Venezuela. Correo electrónico: anamasalazar@gmail.com 
y un incremento significativo de los dienos conjugados en los $\mathrm{HbSC}$, fenotipo severo. Los valores de Hcy, en lo pacientes con HbSS con fenotipos moderado y severo fueron significativamente menores. Los nitritos no mostraron cambios. Este estudio evidencia un aumento de algunos biomarcadores de estrés oxidativo en los genotipos HbSS y HbSC, lo que muestra la importancia de su evaluación en pacientes con SD, para tratar de prevenir complicaciones clínicas mediante el tratamiento con antioxidantes.

\title{
Biomarkers of oxidative stress and their association with the clinical severity of Venezuelan sickle cell patients.
}

Invest Clin 2019; 60 (4): 283-295

Key words: Hemoglobin S; Sickle cell disease; oxidative stress; clinical severity.

\begin{abstract}
Sickle cell disease (SCD) is one of the most common inherited disorders worldwide. It includes a group of hemolytic anemias associated with the presence of hemoglobin S, that are characterized by acute pain episodes and progressive damage of different organs. In Venezuela, SCD frequency ranges between 0 and 7\%. The overproduction of highly oxidizing molecules, known as reactive oxygen and reactive nitrogen species, are part of the pathophysiology and induce a state of oxidative stress that is associated with endothelial dysfunction and tissue damage in SCD. The aim of this work was to evaluate biomarkers of oxidative stress and associated them with the clinical severity of Venezuelan sickle cell patients. In this sense, we evaluated biomarkers related to oxidative stress (serum nitrites, conjugated dienes, thiobarbituric acid reactive substances (TBARS) and serum homocysteine) and were associated with the clinical severity of the patient. Twenty two patients were classified according to their genotype (HbSS, HbSC and HbS $\beta$ tal) and phenotype (mild, moderate and severe). Regarding the lipid peroxidation markers, a significant increase of TBARS was observed in patients with HbSS and severe phenotype $(\mathrm{p}<0.05)$, besides a significant increase of conjugated dienes in HbSC patients with severe phenotype $(\mathrm{p}<0.05)$. The values of homocysteine, in patients with HbSS and moderate and severe phenotypes were significantly lower $(\mathrm{p}<0.05)$. Nitrite values did not show statistically significant changes. This study shows that in Hb SS and SC genotypes an increase in oxidative stress occurs especially in severe phenotypes, demonstrating the importance of evaluating different oxidative stress biomarkers in patients with SCD, in order to prevent endothelial damage and complications of the disease through antioxidant treatments.
\end{abstract}

Recibido: 26-11-2018 Aceptado: 16-10-2019 


\section{INTRODUCCIÓN}

El síndrome drepanocítico (SD) es una entidad clínica asociada a la variante de hemoǵlobina (Hb) S; su herencia es de carácter autosómico recesivo $(1,2)$ y es una de las alteraciones hereditarias más comunes en todo el mundo (1,4-6). Los sujetos homocigotos y dobles heterocigotos para la $\mathrm{HbS}$ se caracterizan por cursar con un proceso hemolítico crónico asociado a la polimerización de la HbS desoxigenada, que conduce a la deformación del glóbulo rojo, siendo este el factor desencadenante de la fisiopatología de la enfermedad $(7,8)$. Tras la polimerización de la $\mathrm{Hb}$, ocurren una serie de eventos que incluyen: a) incremento de la adherencia de los glóbulos rojos al endotelio disfuncional $(9,10)$, b) expresión de moléculas de adhesión $(8,10,11)$, c) liberación de mediadores proinflamatorios, d) activación y reclutamiento de leucocitos (12), e) activación de la coagulación sanguínea, f) hiperproducción de moléculas altamente oxidantes conocidas como especies reactivas de oxígeno (ERO) y especies reactivas de nitrógeno (ERN). Estos procesos moleculares, celulares y biofísicos se combinan para promover el desarrollo de diversos procesos patológicos como inflamación, disfunción endotelial (13-16) y manifestaciones de alteraciones multiorgánicas con crisis dolorosas, complicaciones trombóticas, síndrome torácico agudo, hipertensión pulmonar y accidentes cerebrovasculares, entre otros $(4,8,13-15)$.

Se estima que el 5\% de la población mundial es portadora de alguna mutación en los genes de la $\mathrm{Hb}$, destacando la talasemia y SD $(17,18)$. En Venezuela, la frecuencia del portador de la mutación de la $\mathrm{Hb} \mathrm{S}$ varía entre 0 y 7\% (19-21). La incidencia del SD en conjunto con sus graves complicaciones clínicas, la baja calidad y expectativa de vida, que oscila entre los 42 a 58 años aproximadamente, hace de este síndrome un tema de gran impacto social.

En el SD la elevada producción de ERO y ERN, es causada por diversos mecanismos; tales como: niveles excesivos de hemoglobina libre con acción catalítica sobre las reacciones oxidativas; daño recurrente por la isquemia-reperfusión; estado proinflamatorio crónico y alta oxidación de la $\operatorname{HbS}(8-10,15$, 22-23), lo que puede ocasionar un efecto negativo sobre proteínas, lípidos y ácidos nucleicos. Los eritrocitos de los pacientes suelen exhibir peroxidación lipídica y oxidación de grupos tiólicos. A su vez, los sistemas de defensa antioxidante se ven afectados y/o no son lo suficientemente eficaces para neutralizar la excesiva producción de especies oxidantes, lo que induce los procesos patológicos antes mencionados $(22,23)$. La acumulación de ERO y ERN es una característica relevante en el SD y favorece la hemólisis intravascular, el ciclo isquemia-reperfusión que exacerba los procesos fisiopatológicos y la aparición de complicaciones de la enfermedad, de allí la importancia de realizar estudios de potenciales biomarcadores que permitan dilucidar mecanismos de la enfermedad, para un mejor seguimiento clínico y, a su vez, orientar en la implementación de nuevos tratamientos.

\section{MATERIALES Y MÉTODOS}

\section{Sujetos}

Este estudio incluyó una población de 24 pacientes venezolanos con SD, que acudieron a la consulta de Hematología del Banco Municipal de Sangre del Distrito Capital (BMSDC), mayores de 18 años, que no recibían suplementos con carotenoides, vitaminas E y C. Tampoco recibían hidroxiurea ni quelantes de hierro, al momento de la toma de muestra. Se excluyeron pacientes en periodo de gestación, con enfermedades autoinmunes, cuadros infecciosos recientes, $\mathrm{O}$ que presentaran crisis dolorosas al tomar la muestra. El grupo control estuvo comprendido por 23 personas aparentemente sanas, venezolanas, comparables en edad y sexo al grupo experimental, que acudieron como voluntarios al Laboratorio de Fisiopatología, Sección Coagulación, del Instituto Venezolano de Investigaciones Científicas. 
Esta investigación se realizó bajo los principios de la Declaración de Helsinki y los procedimientos fueron aprobados por el Comité de Bioética del BMSDC. Las muestras de sangre y la información clínica, se recolectaron posterior a la aceptación del consentimiento informado por cada participante.

\section{Muestras de sangre}

Las determinaciones del estudio se realizaron con $10 \mathrm{~mL}$ de sangre venosa de pacientes y controles, las cuales se obtuvieron a través de una punción venosa en el antebrazo. Se colocaron $3 \mathrm{~mL}$ de sangre en un tubo con anticoagulante EDTA $\mathrm{K}_{2}$ para la obtención del plasma y el resto en tubos sin anticoagulante para la obtención del suero.

\section{Parámetros hematológicos}

La cuantificación de $\mathrm{Hb}$, el recuento de glóbulos rojos, hematocrito e índices hematimétricos, se realizaron mediante el autoanalizador Coulter ${ }^{\circledR}$ LH 750 (Bekman Coulter, USA).

Estudio de las fracciones de hemoglobinas y variantes

Las fracciones de $\mathrm{Hb}$ y sus variantes fueron estudiadas por el método de electroforesis capilar, mediante el autoanalizador MiniCap (Sebia, Francia). Las hemoglobinas presentes en cada muestra fueron separadas a través del sistema automatizado de electroforesis capilar, detectada espectrofotométricamente a $415 \mathrm{~nm}$ e identificadas según el patrón electroforético establecido por el fabricante. El control de calidad fue realizado con controles comerciales normales y patológicos a concentraciones conocidas de las diferentes hemoglobinas normales y variantes.

\section{Clasificación de los pacientes}

Los pacientes se agruparon según los siguientes criterios: genotipo y fenotipo clínico. La clasificación genotípica se realizó tomando en cuenta el estudio de las variantes de hemoglobina, posteriormente estos resultados fueron confirmados a través de la secuenciación de los tres exones del gen beta-globina (datos no mostrados).

Con respecto al fenotipo, tomando en cuenta los criterios más utilizados en la literatura $(22,24-26)$ y el de los médicos tratantes de la enfermedad del BMSDC, los pacientes fueron agrupados en los fenotipos leve, moderado o severo, según el número de crisis dolorosas por año $(\mathrm{CD} / \mathrm{a})$ y la presencia de complicaciones ǵraves como accidente cerebro vascular (ACV), síndrome de tórax agudo (STA), complicaciones pulmonares, cardíacas y/o falla renal. El grupo leve incluyó pacientes que presentaron entre $0 \mathrm{y}$ $2 \mathrm{CD} / \mathrm{a}$, el moderado entre 3 y $5 \mathrm{CD} / \mathrm{a}$ y el severo con $\geq 6 \mathrm{CD} / \mathrm{a}$. Aquellos pacientes con alǵuna complicación grave, independiente de las $\mathrm{CD} / \mathrm{a}$ fueron considerados dentro del fenotipo severo.

\section{Dienos conjugados}

Su determinación se realizó mediante el método de Folch (27) y fue medido en un espectrofotómetro de luz ultravioleta (Spectronic, Genesis, USA) a $233 \mathrm{~nm}$; los resultados fueron reportados en $\mu \mathrm{M} / \mathrm{g}$.

\section{TBARS}

Se cuantificaron por el método de Bege y Aust (28). La concentración de los TBARS se calculó por medio del coeficiente de extinción de $1,56 \times 10^{5} \mathrm{M}^{-1} \mathrm{~cm}^{-1} \mathrm{y}$ se expresó en $\mathrm{nmol} / \mathrm{mL}$.

\section{Nitritos séricos}

Se cuantificaron mediante la reacción de Griess (29) en un lector de microplacas de ELISA (Tecan-Sunrise, USA). La concentración de nitritos séricos se obtuvo a partir de una curva de calibración; se utilizó como patrón nitrato de potasio y se expresó en $\mu \mathrm{mol} / \mathrm{L}$.

\section{Homocisteína plasmática total}

La determinación se realizó por el método de García (30), con algunas modificaciones. La separación de los derivados de sal de amonio de ácido 7-Fluorobenzofurazan- 
4-sulfónico (SBD-F) se realizó por medio de una Cromatografía Líquida de Alta Resolución (CLAR) modelo brize binarium (Waters, USA), con una columna C18 Supelco (Sigma-Aldrich, USA), en condiciones isocráticas con tampón $\mathrm{KH}_{2} \mathrm{PO}_{4} 50 \mathrm{mM}, \mathrm{pH}$ 2,3 con $\mathrm{CH}_{3} \mathrm{CN}$ en proporción 98:2. La detección de homocisteína (Hcy) se realizó con un detector fluorescencia que captó la señal del compuesto Hey-SBD-F a 516 nm después de una excitación a una longitud de onda de 386 nm (29) y fue expresado en mM. Para la calibración del método se utilizó un estándar de cisteamina.

\section{ANÁLISIS ESTADÍSTICO}

Los resultados fueron expresados como la media \pm la desviación estándar. El análisis estadístico se realizó por medio de la prueba $\mathrm{t}$ de Student, ANOVA seguido de un test de múltiples comparaciones (Tukey), empleando el software GraphPrisma. El nivel de significancia definido para este estudio fue de un valor de $\mathrm{p}<0,05$ en todos los análisis.

\section{RESULTADOS}

La clasificación por genotipo de los 24 pacientes con SD estudiados se muestra en la Tabla I, de los cuales15 presentaron el genotipo HbSS, 6 pacientes con HbSC y 3 con HbS $\beta$ tal.

En el grupo de pacientes se estimó la frecuencia de transfusiones, hospitalizaciones y crisis dolorosas por año, así como el porcentaje de eventos clínicos que en algún momento comprometieron su vida. De esta forma, se obtuvo una media general de 4 crisis dolorosas y 3 transfusiones por año. El $12 \%$ de la población presentó alguna afección neurológica, un 18\% lesiones articulares y trombóticas, así como un 14\% afecciones renales y ulceraciones en miembros inferiores (Fig. 1).

Los genotipos de los pacientes se agruparon según el grado de severidad de la enfermedad o fenotipo, en leve, moderado y severo. Del total de pacientes con el genotipo HbSS, el 47\% manifestó un fenotipo severo, seguido de un 33\% del moderado y un 20\% del leve. Por su parte, la población con HbSC se aǵrupó en $50 \%$ severo y $50 \%$ leve.

Los parámetros hematológicos se muestran en la Tabla II. Con respecto al grupo control, se observaron diferencias significativas en algunos parámetros, destacándose los pacientes con HbSS, quienes tenían disminuidos la $\mathrm{Hb}$ y el hematocrito (HTO), y aumentados el Volumen Corpuscular medio

TABLA I

DATOS EPIDEMIOLÓGICOS Y HEMOGLOBINA EN GRUPO CONTROL Y PACIENTES CON SD.

\begin{tabular}{|c|c|c|c|c|c|c|c|c|}
\hline \multirow[t]{2}{*}{ Genotipo } & \multirow{2}{*}{$\begin{array}{l}\text { Edad } \\
\text { (años) }\end{array}$} & \multicolumn{2}{|c|}{ Sexo } & \multirow{2}{*}{$\begin{array}{c}\mathrm{Hb} \mathrm{A} \\
(\%)\end{array}$} & \multirow{2}{*}{$\begin{array}{c}\mathrm{Hb} \text { A2 } \\
(\%)\end{array}$} & \multirow[t]{2}{*}{$\mathrm{Hb} \mathrm{F}(\%)$} & \multirow[t]{2}{*}{ Hb S (\%) } & \multirow[t]{2}{*}{ Hb C (\%) } \\
\hline & & $\mathrm{F}$ & $M$ & & & & & \\
\hline $\begin{array}{c}\text { Control } \\
\operatorname{HbAA}(n=23)\end{array}$ & $33 \pm 7$ & 13 & 10 & $97,5 \pm 0,2$ & $2,5 \pm 0,2$ & 0 & & - \\
\hline $\begin{array}{c}\text { Pacientes } \\
\text { HbSS }(n=15) \\
68,2 \%\end{array}$ & $36 \pm 11$ & 11 & 4 & $8,4 \underset{*}{ \pm 17,2}$ & $2,1 \pm 0,5$ & $11,1_{* *}^{ \pm} 8,1$ & $\begin{array}{c}77,9 \pm 16,3 \\
*\end{array}$ & - \\
\hline $\begin{array}{c}\operatorname{HbSC}(n=6) \\
22,7 \%\end{array}$ & $28 \pm 9$ & 3 & 3 & 0 & $3,0 \pm 1,2$ & $2,2 \pm 0,8$ & $\begin{array}{c}52,8 \pm 3,0 \\
*\end{array}$ & $\begin{array}{c}42,0 \pm 2,5 \\
*\end{array}$ \\
\hline $\begin{array}{c}\text { HbS } \beta \text { tal }(\mathrm{n}=3) \\
9,1 \%\end{array}$ & $31 \pm 3$ & 0 & 3 & 0 & $4,2 \pm 0,4$ & $29,6 \pm 6,2$ & $66,3 \pm 5,9$ & - \\
\hline
\end{tabular}

Datos representan la media \pm la desviación estándar y frecuencia absoluta (para la distribución de casos por sexo) "p $<0,05$ vs controles. ${ }^{*} \mathrm{p}<0,05$ vs genotipos de la enfermedad.

Vol. 60(4): $283-295,2019$ 


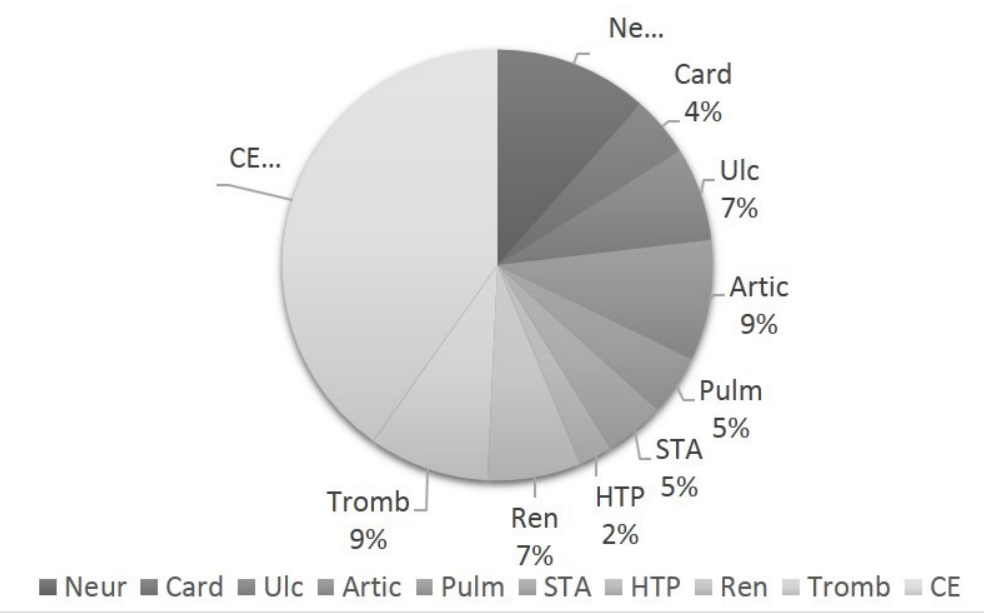

Fig. 1. Complicaciones clínicas del SD. Neur: neurológicas, Card: cardiológicas, Ule: úlceras, Artic: articulares, Pulm: pulmonares STA: síndrome de tórax aǵudo, HTP: hipertensión pulmonar; Ren: Renales, Tromb: Trombosis, CE: Complicaciones en el embarazo.

TABLA II

PARÁMETROS HEMATOLÓGICOS EN GRUPO CONTROL Y PACIENTES CON SD

\begin{tabular}{|c|c|c|c|c|c|}
\hline Genotipos & $\begin{array}{c}\mathrm{Hb} \\
(\mathrm{g} / \mathrm{dL})\end{array}$ & $\begin{array}{l}\text { Hto } \\
(\%)\end{array}$ & $\begin{array}{l}\text { VCM } \\
\text { (fL) }\end{array}$ & $\begin{array}{l}\text { CHCM } \\
(\mathrm{g} / \mathrm{dL})\end{array}$ & $\begin{array}{l}\mathrm{HCM} \\
\text { (pǵ) }\end{array}$ \\
\hline $\begin{array}{c}\text { Control } \\
\text { HbAA } \\
(n=23)\end{array}$ & $13,9 \pm 1,0$ & $41,8 \pm 3,2$ & $89,8 \pm 3,9$ & $33,6 \pm 0,6$ & $30,2 \pm 1,4$ \\
\hline $\begin{array}{l}\text { Pacientes } \\
(\mathrm{n}=22)\end{array}$ & $\underset{*}{9,0 \pm 2,0}$ & $\underset{*}{26,4} \underset{*}{ \pm} 6,6$ & $91,0 \pm 11,1$ & $33,6 \pm 1,0$ & $30,6 \pm 4,2$ \\
\hline $\begin{array}{c}\text { HbSS } \\
(n=15) \\
68,2 \%\end{array}$ & $7,6 \pm \underset{*}{*} 1,5$ & $\underset{*}{21,7} \underset{*}{ \pm} 4,5$ & $\underset{*}{96,8 \pm 12,0}$ & $34,1 \pm 1,2$ & $\begin{array}{c}33,0 \pm 4,1 \\
*\end{array}$ \\
\hline $\begin{array}{l}\text { HbSC } \\
(n=6) \\
22,7 \%\end{array}$ & $12,0 \pm 1,8$ & $36,5 \pm 6,3$ & $\begin{array}{c}76,6 \pm 7,1 \\
*\end{array}$ & $33 \pm 0,7$ & $\begin{array}{c}25,2 \pm 2,2 \\
*\end{array}$ \\
\hline $\begin{array}{c}\text { HbS } \beta \text { tal } \\
(\mathrm{n}=3) \\
9,1 \%\end{array}$ & $\begin{array}{c}11,7 \pm 0,1 \\
*\end{array}$ & $36,6 \pm 0,4$ & $82,9 \pm 6,2$ & $32 \pm 0,2$ & $\begin{array}{c}26,5 \pm 1,9 \\
*\end{array}$ \\
\hline
\end{tabular}

Datos representan la media \pm la desviación estándar. " $\mathrm{p}<0,05$ vs controles.

(VCM) y la Hemoǵlobina Corpuscular media (HCM). La Concentración de Hemoglobina Corpuscular media (CHCM) se encontró disminuida solo en el grupo con HbS $\beta$ tal.

Con respecto al estudio de parámetros de estrés oxidativo, la concentración de dienos conjugados, en relación al control $(9,49 \pm 2,56 \mu \mathrm{M} / \mathrm{g})$, los pacientes con $\mathrm{SD}$ presentaron valores significativamente mayores en el grupo $\operatorname{HbSC}(16,43 \pm 6,57$ $\mu \mathrm{M} / \mathrm{g} ; \mathrm{p}<0,05)$, encontrándose siǵnificancia en el fenotipo severo $(21,07 \pm 3,71 \mu \mathrm{M} / \mathrm{g}$; $\mathrm{p}<0,05)$ (Fig. 2).

La concentración de los TBARS, en relación al grupo control $(0,77 \pm 0,18 \mathrm{nmol} / \mathrm{mL})$ se encontró significativamente más elevada $(\mathrm{p}<0,05)$ en los pacientes HbSS con fenotipo severo $(1,05 \pm 0,22 \mathrm{nmol} / \mathrm{mL})$ (Fig. 3). 


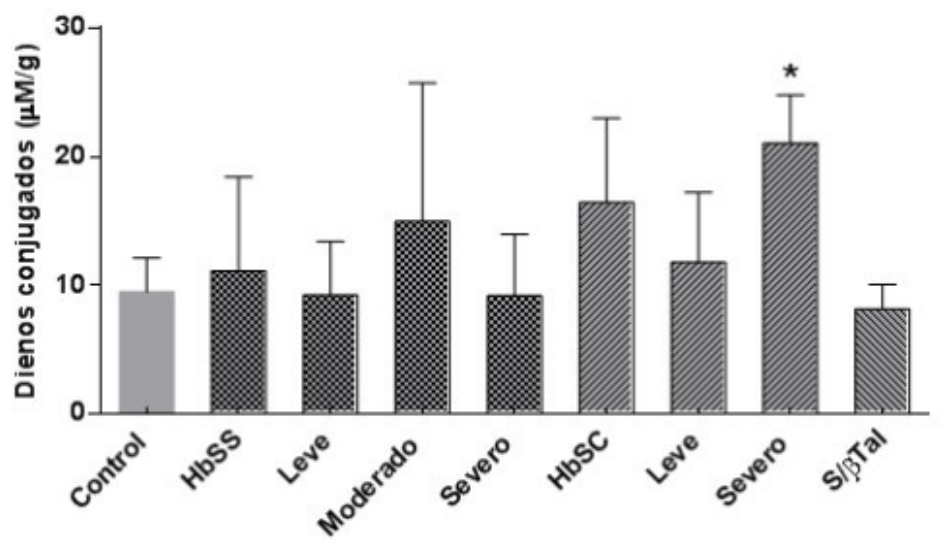

Fig. 2. Concentración de dienos conjugáados $\mu \mathrm{M} / \hat{\mathrm{g}}$ ), en controles (HbAA, $\mathrm{n}=23$ ) y pacientes con SD, aǵrupados por: genotipo $\operatorname{HbSS}(n=15)$ y sus fenotipos leve $(n=3)$, moderado $(n=5)$ y severo $(n=7)$; genotipo HbSC $(n=6)$ y sus fenotipos leve $(n=3)$ y severo $(n=3)$ y genotipo HbS/ $\beta$ tal $(n=3)$. Los resultados representan la media \pm la desviación estándar. * $\mathrm{p}<0,05$.

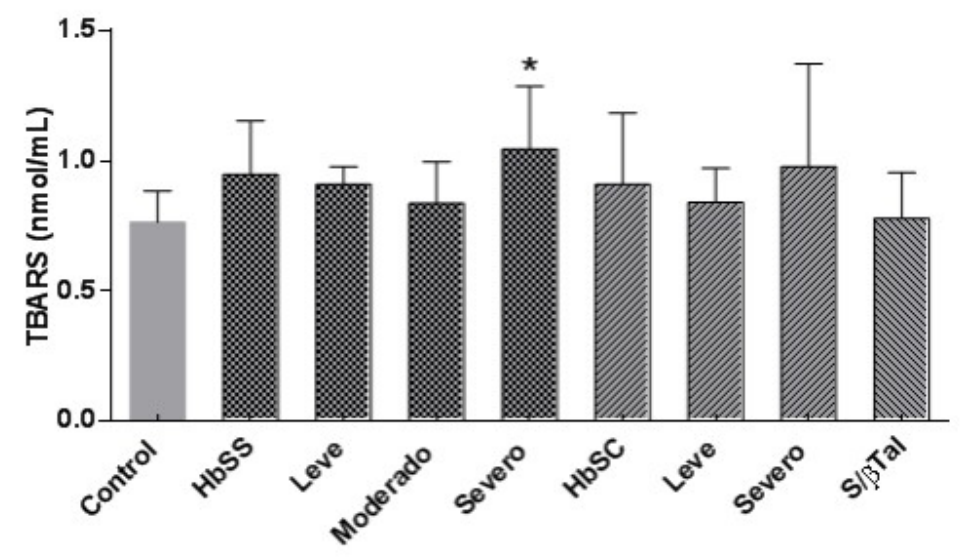

Fig. 3. Concentración de TBARS ( $\mathrm{nmol} / \mathrm{mL})$, en controles $(\mathrm{HbAA}, \mathrm{n}=23)$ y pacientes con SD, agrupados por: genotipo HbSS $(n=15)$ y sus fenotipos leve $(n=3)$, moderado $(n=5)$ y severo $(n=7)$; genotipo HbSC $(n=6)$ y sus fenotipos leve $(n=3)$ y severo $(n=3)$ y genotipo $\mathrm{HbS} / \beta$ tal $(n=3)$. Los resultados representan la media \pm la desviación estándar. " $\mathrm{p}<0,05$.

La concentración de Hey, en relación al grupo control $(9,43 \pm 1,80 \mu \mathrm{M})$, fue significativamente menor $(\mathrm{p}<0,05)$ en los pacientes $\operatorname{HbSS}(6,97 \pm 2,20 \mu \mathrm{M})$, con los fenotipos moderado y severo $(7,26 \pm 1,86$ y 5,07 $\pm 0,74 \mu \mathrm{M}$, respectivamente) (Fig. 4).

La concentración de nitritos séricos, en todos los genotipos y fenotipos evaluados fue similar a la del grupo control $(22,84 \pm 6,63$ $\mu \mathrm{mol} / \mathrm{L}),(\mathrm{p}>0,05)$.

\section{DISCUSIÓN}

Reportes de la Organización Mundial de la Salud (OMS) estiman que aproximadamente 5\% de la población mundial es portadora de genes causantes de alteraciones de la Hb y cada año nacen más de 300.000 niños con hemoglobinopatías graves, siendo la más frecuente el SD $(11,31)$. Los individuos con enfermedad drepanocítica se caracteri- 


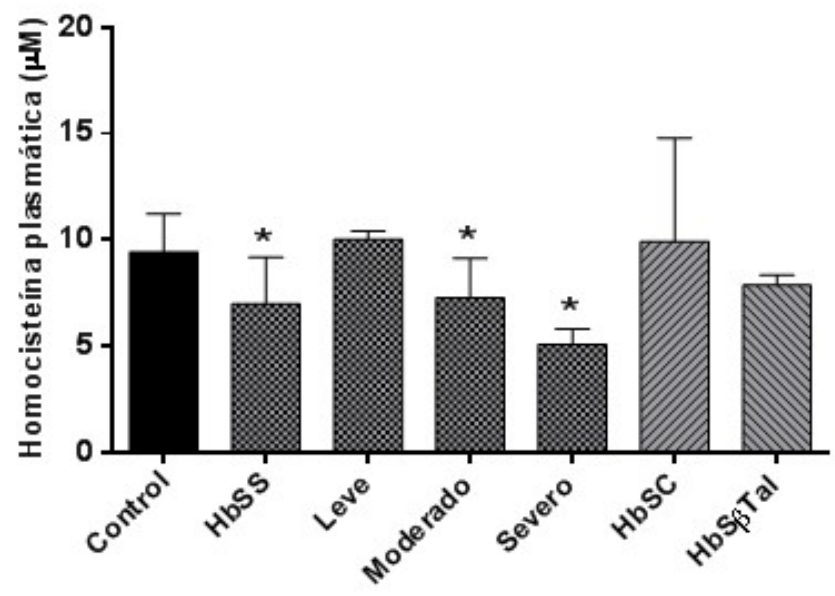

Fig. 4. Concentración de homocisteína en pacientes con SD, asociación con genotipo HbSS ( $\mathrm{n}=15)$ y sus fenotipos leve $(n=3)$, moderado $(n=5)$ y severo $(n=7)$ y los genotipos $\operatorname{HbSC}(n=6)$ y $\operatorname{HbS} / \beta \operatorname{tal}(n=3)$. Los resultados representan la media \pm la desviación estándar * $\mathrm{p}<0,05$.

zan por manifestar cuadros de anemia y una gran diversidad de complicaciones clínicas que limitan sus condiciones y calidad de vida. Las altas tasas de morbilidad se deben a que gran parte de esta población tiene un estilo de vida limitado por los múltiples factores genéticos y ambientales que fomentan el desarrollo de su fisiopatología (32).

En el grupo de pacientes venezolanos con SD incluidos en este estudio, se observó la HbSS como el genotipo más frecuente, seguido de HbSC y HbS $\beta$ tal; estos datos concuerdan con un estudio retrospectivo en Venezuela (21) en el cual se identificaron genéticamente a más de 1.000 pacientes con SD, durante un periodo de casi 20 años. De igual manera se reportaron resultados similares en una revisión de hemoǵlobina $\mathrm{S}$ en Venezuela (20). La distribución genotípica de la enfermedad ha sido reportada en pacientes de la isla de Cuba (32) y en pacientes del continente americano (33).

Se ha descrito que los pacientes con los genotipos HbSS y HbS $\beta$ tal desarrollan estados de anemia crónica más severos en comparación a los heterocigotos compuestos HbSC $(33,34)$. En el presente trabajo se evidenció una menor concentración de $\mathrm{Hb}$, en los pacientes con genotipo HbSS, con res- pecto al control y a los otros genotipos. En comparación al grupo con genotipo HbSC, los pacientes HbS $\beta$ tal mostraron anemia leve. Resultados similares fueron reportados previamente (32), incluyendo a pacientes con HbSC procedentes de diversos países de África (35). Los pacientes con genotipos HbSS y HbS $\beta$ tal generalmente tienen un proceso hemolítico más severo y un tiempo de vida media menor de sus eritrocitos, debido a que la mayor parte de su $\mathrm{Hb}$ es $\mathrm{HbS}$, a diferencia de los pacientes con genotipos $\mathrm{HbSC}$, siendo la hemoglobina $C$ una variante que genera una hemoǵlobinopatía menos severa y que no polimeriza. Sin embargo, en los pacientes HbSC se observaron alteraciones importantes en los parámetros de estrés oxidativo y este podría ser un factor determinante para la presentación de complicaciones de la enfermedad.

Los criterios empleados para la clasificación de los pacientes con SD en los fenotipos severo, moderado y leve, fueron similares a los utilizados por Rusanova y su grupo de trabajo (22), quienes consideraron el número de crisis dolorosas por año y la aparición de complicaciones clínicas como criterios para establecer los grados de severidad. La aparición de diversas manifestaciones clíni- 
cas en el SD incrementa el riesgó de hospitalización $(1,12,31,36)$. Las complicaciones que llevan a la hospitalización están asociadas a los permanentes estados de hemólisis y a los procesos vaso-oclusivos (36), eventos que pueden estar relacionados a un mayor estrés oxidativo y a alteraciones hemostáticas $(36,37)$. En este sentido, en una revisión asociada al SD y el sistema hemostático (38), encontraron alteraciones en diferentes componentes de la hemostasia; entre estas; las asociadas a la función coagulante, que incluyen aumento de la expresión de proteínas de adhesión, incremento de la expresión del factor tisular (FT) en micropartículas derivadas de varios tipos de células y disminución de las proteínas $\mathrm{C}$ y S, que favorecen la aparición de manifestaciones trombóticas.

En el SD, la formación de ERO y ERN están asociadas a diversos procesos fisiopatológicos de la enfermedad, incluyendo la auto-oxidación de la HbS en condiciones de hipoxia $(7,8)$, lo que induce la producción de grandes cantidades de anión superóxido $\left(\mathrm{O}_{2}^{-}\right)$, a partir del cual se generan otras ERO como el peróxido de hidrógeno $\left(\mathrm{H}_{2} \mathrm{O}_{2}\right)$. Adicionalmente, los reiterados procesos de hemólisis, liberan hemoglobina al plasma y saturación de las proteínas transportadoras, lo que induce la reacción del $\mathrm{H}_{2} \mathrm{O}_{2}$ con hierro del grupo hemo en estado ferroso $\left(\mathrm{Fe}^{2+}\right)$, produciendo anión hidroxilo $(\mathrm{OH})$ a través de la reacción de Fenton, un potente agente oxidante. No obstante, el ON puede evitar dicha formación al ejercer un rol antioxidante, siempre y cuando la cantidad de ERO no exceda su concentración, dado que el ON puede reaccionar con las ERO y formar ERN (6, 12, 14,23).

Se ha descrito que los radicales libres, como el anión superóxido, son capaces de reaccionar con el ON y generar especies reactivas como el peroxinitrito (ONOO-), las cuales están involucradas en la peroxidación lipídica y promueve el daño oxidativo en órganos y tejidos (39). Asimismo, se ha reportado en individuos homocigotos con SD que las ERN pueden favorecer la hemólisis intra- vascular, lo que conduce a la liberación de arginasas y de $\mathrm{Hb}$ hacia la circulación, que contribuyen con la disminución de la biodisponibilidad del ON. Sin embargo, el exceso de ERO y ERN pueden incrementar la biodisponibilidad del ON, ya que estos pueden inducir la expresión de la enzima iNOS y favorecer la síntesis de ON (40-42). Además, se ha descrito en el SD un estado de disfunción endotelial similar a la descrita en individuos con enfermedades arteriales coronarias y ateroesclerosis, caracterizada por una respuesta limitada a la inhibición de NOS, lo que finalmente contribuye a la vasculopatía que se desarrolla en el SD (43).

En la presente investigación no se encontraron diferencias significativas entre pacientes y controles en relación al ON. Esto puede deberse a que los mecanismos mencionados que modulan la biodisponibilidad de ON se encuentran en un aparente equilibrio y pueden compensarse entre sí, sin observarse una variación en los valores normales de ON $(42,44,45)$. Asimismo, los resultados obtenidos pueden deberse a que los individuos con el genotipo HbSC y HbS $\beta$ tal presentaron concentraciones de Hb más elevados que el genotipo HbSS, lo que puede indicar un proceso hemolítico más leve en estos pacientes, que a su vez disminuye la liberación a la circulación sanguínea de arginasas y $\mathrm{Hb}$ que puedan modular negativamente la biodisponibilidad del ON.

$\mathrm{El}$ incremento de las ERO y ERN derivadas de las diversas fuentes en el SD, ocasionan la oxidación de los ácidos grasos poliinsaturados presentes en la membrana celular de los eritrocitos y conduce a la formación de productos de la peroxidación lipídica como son los dienos conjugados, hidroxiperóxidos y malondialdehído (MDA) (46). En el presente estudio, la peroxidación lipídica se determinó mediante dos biomarcadores, dienos conjugados como representante de la fase inicial de este proceso, los cuales surgen tras el reordenamiento de los dobles enlaces del radical lipídico formado por acción de la especie reactiva iniciadora, y MDA mediante 
la determinación de TBARS, uno de los productos finales de la peroxidación lipídica, el cual se forma principalmente por la descomposición de los peróxidos de los ácidos grasos con tres o más dobles enlaces y también durante el metabolismo de ácido araquidónico para la síntesis de prostaǵlandinas (47).

Los valores de los dienos conjugados, en relación a los controles, se observaron más elevados en los pacientes con genotipo HbSC, en el fenotipo severo; sin embargo, este marcador no se encontró elevado en los pacientes SS con fenotipo severo. Cabe destacar que la fisiopatología del SD es muy compleja y participan muchas variables genéticas, bioquímicas e inmunológicas derivadas de los procesos fisiopatológicos y del estado inflamatorio crónico, así como cualquier otra patología subyacente que puedan presentar los pacientes como hipertensión, diabetes, dislipidemias, entre otras, así como el daño orgánico producido por las complicaciones de la enfermedad. Este hallazgo en el genotipo HbSC puede estar relacionado con el mayor número de complicaciones graves que manifiestan este grupo de los pacientes, presentando un mayor estado de estrés oxidativo que puede inducir más complicaciones y empeorar su condición clínica. Este marcador ha sido utilizado en patologías de origen endocrino (46), enfermedad coronaria (47) y en preeclamsia (48), donde se ha observado que el incremento de la peroxidación lipídica, específicamente de dienos conjugados, es un factor determinante en la fisiopatología de estas enfermedades.

La evaluación de la etapa final del proceso de peroxidación lipídica, a través de TBARS, demostró que los pacientes homocigotos para la HbSS del fenotipo severo tienen un mayor grado de oxidación, lo que permite relacionar la severidad con el grado de peroxidación lipídica. Otros investigadores han obtenido resultados similares de TBARS en el SD $(22,49)$.

La hiperhomocisteinemia está asociada con la deficiencia de folatos, vitaminas
$\mathrm{B}_{6} \mathrm{y}_{12}$. En diversos estudios el incremento de la concentración plasmática de Hey se ha asociado con complicaciones vasculares $(50,51)$. No obstante, en esta investigación, con respecto al control, en ninguno de los genotipos del SD se observaron aumentos significativos de Hey; por el contrario, se encontró una disminución significativa en los casos con genotipo HbSS y fenotipo moderado. Podemos relacionar estos resultados con un estudio realizado en pacientes con SD en Curazao (52). Si bien durante el presente estudio no se determinó la concentración del ácido fólico ni de las vitaminas $\mathrm{B}_{12} \mathrm{y}_{6}$, se puede inferir que la concentración plasmática de Hcy de los pacientes incluidos en el presente estudio puede estar relacionada al hecho de que todos tenían indicada una terapia con suplemento de ácido fólico, adicionalmente el 32\% consumía también complejo de vitamina $B$.

Los hallazgos del presente estudio, sugieren un mayor estrés oxidativo en los pacientes con SD con fenotipo severo, lo que cual favorece la disfunción endotelial, la vasculopatía y exacerba el proceso fisiopatológico que involucra hemólisis crónica y vaso-oclusión, factores que promueven la aparición de complicaciones propias de la enfermedad. El estudio de parámetros relacionados al estrés oxidativo aporta a la evaluación de los diferentes de grupos de pacientes y pueden permitir un mejor pronóstico de la evolución y la presentación de complicaciones de la enfermedad. Esta investigación es pionera en el estudio de peroxidación lipídica en el SD. Para ampliar la información obtenida, se sugiere continuar con la evaluación del estado de estrés oxidativo en pacientes venezolanos con SD y su asociación con la enfermedad. Estos resultados podrían ayudar a la implementación de nuevas estrategias terapéuticas, asociadas con la disminución del estrés oxidativo como una medida de prevención de complicaciones clínicas y, de esta manera, mejorar la calidad de vida de estos pacientes. 


\section{AGRADECIMIENTOS}

Los autores desean mostrar su agradecimiento a la MSc. Zoila Carvajal del IVIC; a todo el personal de la consulta de hematología, del Laboratorio de Hematología y el Laboratorio de Hemoǵlobinopatías del Banco Municipal de Sangre del Distrito Capital; como también a la Dra. Fátima Garcés de la Universidad Central de Venezuela, por el apoyo téenico y asesoramiento recibidos.

Este estudio ha sido financiado por el Instituto Venezolano de Investigaciones Científicas (IVIC) y por el Banco Municipal de Sangre del Distrito Capital (BMSDC).

\section{REFERENCIAS}

1. Quintero M, Hernández A. Anemia de células falciformes. Revista Gastrohnup 2012; 14 (2)1: S27-S35.

2. Kato G, Piel F, Reid C, Gaston M, OheneFrempong K, Krishnamurti L, Smith W, Panepinto J, Weatherall D, Costa F, Vichinsky E. Sickle cell disease. Nat Rev Dis Primers 2018; 4: 18010.

3. Villaescusa B. Carest (Asociación Caribeña para Investigadores en Drepanocitosis y Talasemia): un logro caribeño que abre mayores oportunidades de cooperación. Revista cubana de hematología, inmunología, hemoterapia 2014; 30:95-97.

4. Ruiz A, Briceño O, Arteaga M, Plumacher Z, González M, Quintero, M. Anemia hemolítica hereditaria y sobrecarga de hierro. Vitae 2013; 53:1-8.

5. Rees D, Williams T, Gladwin. Sickle cell disease. Seminar 2010; 376: 2018-2031.

6. Kohne E. Hemoǵlobinopathies. DtschArzteblInt 2011; 108: 532-540.

7. Steinberg M. Sickle cell anemia, the first molecular disease: overview of molecular etiology, pathophysiology, and therapeutic approaches. Scientific World Journal 2008; 8:1295-1324.

8. Barabino G, Platt M, Kaul D. Sickle Cell Biomechanics. Annu Rev Biomed 2010; 12: 345-367.

9. Hobbel R, Morgan W, Eaton J, Hedlund B. Accelerated autooxidation and heme loss due to instability of Sickle hemoǵlobin. Proc Natl Acad Sci USA 1988; 85:237-241.

10. Mack A, Kato G. Sickle cell disease and nitric oxide: a paradigm shift? Int $\mathrm{J}$ Biochem Cell Biol 2006; 38:1237-1243.

11. Campo M, Fortún P, Fortún C, Hernández J. Fisiopatología de la vaso-oclusión en la drepanocitosis. Rev Ciencias Médicas 2009;13: 80-89.

12. Conran N, Franco-Penteado C, Costa F. Newer aspects of the pathophysiology of sickle cell disease vaso-occlusion. Hemoglobin 2009; 33:1-16.

13. Sundd P, Gladwin M, Novelli E. Pathophysiology of Sickle Cell Disease. Annu Rev Pathol 2019; 14:263-292.

14. Odièvre $M$, Verger $E$, Silva A, Elion $J$. Pathophysiological insights in sickle cell disease. Indian J Medical Res 2011; 134:532537.

15. Wood K, Granger N. Sickle cell disease: role of reactive and nitrogen metabolites. Clin Exp Pharmacol Physiol 2007; 34:926-932.

16. Rees D, Gibson J. Biomakers in sickle cell disease. Br J Haematol. 2012; 156:433-434.

17. Organización Mundial de la Salud. Drepanocitosis y otras hemoǵlobinopatías. Notas descriptivas 2011; 308.

18. Organización Mundial de la Salud. Anemia falciforme. Informe de la Secretaría de la 59 a Asamblea Mundial de la Salud. 2006.

19. García O, Chacín M, Bravo M, Gómez G, Montilla S, Merzón R, de Donato M, Castillo O, Arends A. Diaǵnóstico de hemoǵlobinopatías a partir de sangre del talón de recién nacidos en diferentes centros hospitalarios de Venezuela. Ann Pediatr 2009; 71: 314-318.

20. Salazar-Lugo R. La hemoglobina $S$ en la población venezolana. Invest Clin 2004; 45 : 175-183.

21. Arends A, Chacín M, Bravo M, Montilla S, Guevara J, Velásquez D, García G, Álvarez M, Castillo O. Hemoǵlobinopatías en Venezuela. Interciencia 2007; 32: 516-521.

22. Rusanova I, Escames G, Cossio G, Borance R, Moreno B, Chahboune M, López L, Díez T, Acuña-Castroviejo D. Oxidative stress status, clinical outcome, and $\beta$-globin gene cluster haplotypes in pediatric patients with Sickle cell disease. Eur J Hematology 2010; 85: 529-537. 
23. Nolfi-Donegan D, Pradhan-Sundd T, Pritchard K, Hillery C. Redox Signaling in Sickle Cell Disease. Curr Opin Physiol 2019; 9: 26-33.

24. Falusi A, Olatunji P. Effects of alpha thalassaemia and haemoglobin $\mathrm{F}$ level on the clinical severity of sickle cell anaemia. Eur J Hematol 1994; 52: 13-15.

25. Diop S, Thiam D, Cisse M, Toure-Fall A, Fall K, Diakhate L. New results in clinical severity of homozygous sickle cell anemia in Dakar, Senegal. Hematol Cell Ther 1999; 41:217-221.

26. Rodríguez D. Drepanocitosis: Influencia de factores de riesgó trombogénico en la severidad de crisis vaso-oclusivas. Tesis de Especialidad en Hematología, Facultad de Medicina-UCV. 2001.

27. Folch J, Lees M, Sloane-Stanley G. A simple method for the isolation and purification of total lipids from animal tissues. J Biol Chem 1957; 226: 497-509.

28. Bege J, Aust S. Microsomal Lipid Peroxidation. Methods in Enzymology 1978; 52: 302-310.

29. Griess, J. P. Bemerkungen zu der Abhandlung der HH. Weselsky und Benedikt Ueber einige Azoverbindungen. Deutsch Chem Ges 1879; 12: 426.

30. García A, Apitz-Castro R. Plasma total homocysteine quantification: an improvement of the classical high-performance liquid chromatographic method with fluorescence detection of the thiol-SBD derivatives. J Chrom B Analyt Technol Biomed Life Sei 2002; 779:359-363.

31. Granger D, Korthuis R. Physiologic mechanisms of postischemic tissue injury. Annu Rev Physiol 1995; 57:311-332.

32. Machín S, Guerra T, Svarch E, Espinosa E, Mesa J, Dorticós E, Ternblom A, González A, Nuñez A, Wade M. Morbiletalidad en pacientes adultos con drepanocitosis. Rev Cubana Hematol Inmunol Hemoter 2004; 20 (2).

33. Saraf L, Molokie R, Nouraie M, Sable C, Luchtman-Jones L, Ensing G, Campbell A, Rana S, Niu X, Machado R, Gladwin M, Gordeuk V. Differences in the clinical and genotypic presentation of sickle cell disease around the world. Paediatr Respir Rev 2014; 15: 4-12.
34. Asociación Venezolana de Drepanocitosis y Talasemias. Guía de práctica clínica en enfermedad drepanocítica 2013. Caracas: Asociación Venezolana de Drepanocitosis y Talasemias; 2013.

35. Lionnet F, Hammoudi N, Stankovic K, Avellino V, Grateau G, Girot R, Haymann J. Hemoǵlobin sickle cell disease complications: a clinical study of 179 cases. Haematologica 2012; $97(8), 1136-1141$.

36. Wood K, Hsu L, Gladwin M. Sickle cell disease vasculopathy: A state of nitric oxide resistance. Free Radic Biol Med 2008; 44:1506-1528.

37. Pawlinski R, Sparkenbaugh E. Prothrombotic aspects of sickle cell disease. J Thromb Haemost 2017; 15:1307-1316.

38. Higuera D, Bravo M, Guerrero B. Alteraciones de la hemostasia en el síndrome drepanocítico. Invest Clin 2014; 55: 173 - 184.

39. Maeda, H, Akaike T. Nitric oxide and oxyǵen radicals in infection, inflammation, and cancer. Biochemistry 1998; 63, 854865 .

40. Escames G, Khaldy H, León J, Gonzalez L, Acuña-Castroviejo D. Changes in iNOS activity, oxidative stress and melatonin levels in hypertensive patients treated with lacidipine. J Hypertens 2004; 22:626-635.

41. Aslan M, Canatan D. Modulation of redox pathways in neutrophils from sickle cell disease patients. Exp Hematol 2008; 36:1535-1544.

42. Akinsheye I, Klings E. Sickle cell anemia and vascular dysfunction: the nitric oxide connection J Cell Physiol 2010; 224: 620625 .

43. Gladwin M, Kato G. Cardiopulmonary complications of sickle cell disease: role of nitric oxide and hemolytic anemia. Hematology Am Soc Hematol Educ Program. $2005 ; 51-7$.

44. Wood K, Granger N Sickle cell disease: Role of reactive oxygen and nitrogen metabolites. Clin Exp Pharmacol Physiol 2007; 34, 926-932.

45. Elias D, Rocha L, Cavalcante M, Pedrosa A, Justino I, Gonçalves R. Correlation of low levels of nitrite and high levels of fetal hemoglobin in patients with sickle cell disease at baseline. Rev Bras Hematol Hemoter 2012; 34:265-269. 
46. Kolesnikova L, Semyonova N, Grebenkina L, Darenskaya M, Suturina L, Gnusina S. Integral indicator of oxidative stress in human blood. Bull Exp Biol Med 2014; 157 : 715-717.

47. Göçmen A, Gümüşlü S, Semiz E. Association between paraoxonase-1 activity and lipid peroxidation indicator levels in people living in the Antalya regiion with angiographically documented coronary artery disease. Clin Cardiol 2004; 27: 426-430.

48. Abad C, Teppa-Garrán A, Proverbio T, Piñero S, Proverbio F, Marín R. Effect of magnesium sulfate on the calcium-stimulated adenosine triphosphatase activity and lipid peroxidation of red blood cell membranes from preeclamptic women. Biochem Pharmacol 2005; 70: 1634-1641.

49. Bhagat S, Patra P, Thakur A. Association of inflammatory biomarker C-reactive protein, lipid peroxidation and antioxidant capacity marker with HbF level in Sickle cell disease patients from Chattisgarh. Indian J Clin Biochemistry 2012; 27: 394-399.
50. Blanco F, Deulofeu, R. Vilaseca A, Chacon P, Dulí, E. Determinación de homocisteína en plasma: metabolismo, metodología, interpretación de resultados y papel en la evaluación del riesǵo vascular. Med Clin (Bare) 2005; 124: 544-553.

51. Vila N, Deulofeu R, Chamorro A, Piera C. Concentraciones plasmáticas de homocisteína en pacientes con infarto cerebral isquémico. Med Clin (Barc) 1998; 110: 605608.

52. Fey P, Van der D, Schnog J, Brouwer J, Velvis $\mathrm{H}$. Elevated homocysteine levels indicate suboptimal folate status in pediatric Sickle cell patients. Am J Hematoloǵy 1998; 59: 192-198. 